\title{
Commentary/Introduction for "Debate on Insulin vs. Non-insulin Use in the Hospital Setting" Articles
}

\author{
Amisha Wallia ${ }^{1}$ - Jane Jeffrie Seley ${ }^{2}$ \\ Published online: 26 July 2019 \\ (C) Springer Science+Business Media, LLC, part of Springer Nature 2019
}

Since 2005, several new classes of diabetes (DM) medications have become available, changing treatment paradigms involving both oral and injectable therapies. These medications include glucagon-like peptide (GLP-1) receptor agonists, dipeptidyl peptidase 4 (DPP-4) inhibitors, and sodium glucose co-transporter (SGLT2) inhibitors. While national and international guidelines have addressed the placement of these medications in the outpatient setting, this has been more difficult in the inpatient setting. Reasons include lack of robust data in this clinical care setting, potential contraindications with other medications commonly used in the hospital setting, tolerability when acutely ill, and operational barriers such as potential increases in cost. At the same time, data on other DM agents has recently been brought to light for certain subpopulations of inpatients. As these studies add to the literature in the inpatient setting, knowledge of the potential risks, benefits, and practicality of use of these non-insulin agents is a subject of debate. In an effort to offer our readers some guidance, we have invited two leading experts in the field to evaluate the available evidence and elucidate the pros and cons of various pharmacologic strategies. We hope this debate and collegial discussion will help guide your clinical practice decisions in the future.

Publisher's Note Springer Nature remains neutral with regard to jurisdictional claims in published maps and institutional affiliations.
Amisha Wallia

a-wallia@fsm.northwestern.edu

Jane Jeffrie Seley

janeseley@nyp.org

1 Northwestern University Feinberg School of Medicine, Suite 530, 645 N. Michigan Avenue, Chicago, IL 60611, USA

2 Division of Endocrinology, Diabetes \& Metabolism, New York-Presbyterian Hospital/Weill Cornell Medicine, Box 55, 525 East 68 St, Rm F2025, New York, NY 10065, USA 